\title{
Dissection of three quantitative trait loci for grain size on the long arm of chromosome 10 in rice (Oryza sativa L.)
}

\author{
Yujun Zhu ${ }^{1,2}$, Zhichao Sun ${ }^{1}$, Xiaojun Niu ${ }^{1}$, Jiezheng Ying ${ }^{1}$, Yeyang Fan ${ }^{1}$, Tongmin Mou ${ }^{2}$, Shaoqing Tang ${ }^{\text {Corresp., } 1}$, \\ Jieyun Zhuang ${ }^{\text {Corresp. } 1}$ \\ ${ }^{1}$ State Key Laboratory of Rice Biology and Chinese National Center for Rice Improvement, China National Rice Research Institute, Hangzhou, China \\ State Key Laboratory of Crop Genetic Improvement and National Center of Plant Gene Research, Huazhong Agricultural University, Wuhan, China \\ Corresponding Authors: Shaoqing Tang, Jieyun Zhuang \\ Email address: tangshaoqing@caas.cn, zhuangjieyun@caas.cn
}

Background. Thousand grain weight is a key component of grain yield in rice, and a trait closely related to grain length and grain width that are important traits for grain quality. Causal genes for 16 quantitative trait loci (QTL) affecting these traits have been cloned, but more QTL remain to be characterized for establishing a genetic regulating network. A QTL controlling grain size in rice, qGS10, was previously mapped in the interval RM6100-RM228 on chromosome 10. This study aimed to delimitate this QTL to a more precise location.

Method. A total of 12 populations were used. The ZC9 population comprised $203 \mathrm{~S}_{1: 2}$ families derived from a residual heterozygous $(\mathrm{RH})$ plant in the $\mathrm{F}_{9}$ generation of the indica rice cross Teqing/IRBB52, segregating the upper region of RM6100-RM228 and three more regions on chromosomes 1, 9 and 11. The Ti52-1 population comprised $171 \mathrm{~S}_{1}$ plants derived from one $\mathrm{RH}$ plant in $\mathrm{F}_{7}$ of Teqing/IRBB52, segregating a single interval that was in the lower portion of RM6100-RM228. The other ten populations were all derived from Ti52-1, including five $S_{1}$ populations with sequential segregating regions covering the target region and five near isogenic line (NIL) populations maintaining the same segregating pattern. QTL analysis for 1000-grain weight, grain length and grain width was performed using QTL IciMapping and SAS procedure GLM.

Result. Three QTL were separated in the original qGS10 region. The $q G L 10.1$ was located in the upper region RM6704-RM3773, shown to affect grain length only. The qGS10.1 was located within a 207.1-kb interval flanked by InDel markers Te20811 and Te21018, having a stable and relatively high effect on all the three traits analyzed. The qGS10.2 was located within a 1.2-Mb interval flanked by simple sequence repeat markers RM3123 and RM6673. This QTL also affected all the three traits but the effect was inconsistent across different experiments. QTL for grain size were also detected in all the other three segregating regions.

Conclusion. Three QTL for grain size that were tightly linked on the long arm of chromosome 10 of rice were separated using NIL populations with sequential segregating regions. One of them, qGS10.1, had a stable and relatively high effect on grain weight, grain length and grain width, providing a good candidate for gene cloning. Another QTL, qGS10.2, had a significant effect on all the three traits but the effect was inconsistent across different experiments, providing an example of genotype-byenvironmental interaction. 
1 Dissection of three quantitative trait loci for grain size

2 on the long arm of chromosome 10 in rice (Oryza

3 sativa L.)

4 Yujun Zhu ${ }^{1,2}$, Zhichao Sun ${ }^{1}$, Xiaojun Niu ${ }^{1}$, Jiezheng Ying ${ }^{1}$, Yeyang Fan ${ }^{1}$, Tongmin Mou $^{2}$,

5 Shaoqing Tang ${ }^{1}$, Jieyun Zhuang ${ }^{1}$

$6{ }^{1}$ State Key Laboratory of Rice Biology and Chinese National Center for Rice Improvement,

7 China National Rice Research Institute, Hangzhou 310006, China.

$8{ }^{2}$ State Key Laboratory of Crop Genetic Improvement and National Center of Plant Gene

9 Research (Wuhan), Huazhong Agricultural University, Wuhan 430070, China

10

11 Corresponding Author:

12 Jieyun Zhuang

13359 Tiyuchang Road, Hangzhou, Zhejiang, 310006, China

14 Email address: zhuangjieyun@caas.cn

15 Shaoqing Tang

16359 Tiyuchang Road, Hangzhou, Zhejiang, 310006, China

17 Email address: tangshaoqing@caas.cn

18 


\section{Abstract}

Background. Thousand grain weight is a key component of grain yield in rice, and a trait closely related to grain length and grain width that are important traits for grain quality. Causal genes for 16 quantitative trait loci (QTL) affecting these traits have been cloned, but more QTL remain to be characterized for establishing a genetic regulating network. A QTL controlling grain size in rice, $q$ GS10, was previously mapped in the interval RM6100-RM228 on chromosome 10 . This study aimed to delimitate this QTL to a more precise location.

Method. A total of 12 populations were used. The ZC9 population comprised $203 \mathrm{~S}_{1: 2}$ families derived from a residual heterozygous $(\mathrm{RH})$ plant in the $\mathrm{F}_{9}$ generation of the indica rice cross Teqing/IRBB52, segregating the upper region of RM6100-RM228 and three more regions on chromosomes 1, 9 and 11 . The Ti52-1 population comprised $171 \mathrm{~S}_{1}$ plants derived from one $\mathrm{RH}$ plant in $\mathrm{F}_{7}$ of Teqing/IRBB52, segregating a single interval that was in the lower portion of RM6100-RM228. The other ten populations were all derived from Ti52-1, including five $\mathrm{S}_{1}$ populations with sequential segregating regions covering the target region and five near isogenic line (NIL) populations maintaining the same segregating pattern. QTL analysis for 1000-grain weight, grain length and grain width was performed using QTL IciMapping and SAS procedure GLM.

Result. Three QTL were separated in the original $q G S 10$ region. The $q G L 10.1$ was located in the upper region RM6704-RM3773, shown to affect grain length only. The qGS10.1 was located within a 207.1-kb interval flanked by InDel markers Te20811 and Te21018, having a stable and relatively high effect on all the three traits analyzed. The $q G S 10.2$ was located within a 1.2-Mb interval flanked by simple sequence repeat markers RM3123 and RM6673. This QTL also affected all the three traits but the effect was inconsistent across different experiments. QTL for grain size were also detected in all the other three segregating regions.

Conclusion. Three QTL for grain size that were tightly linked on the long arm of chromosome 10 of rice were separated using NIL populations with sequential segregating regions. One of them, $q$ GS10.1, had a stable and relatively high effect on grain weight, grain length and grain width, providing a good candidate for gene cloning. Another QTL, $q$ GS10.2, had a significant effect on all the three traits but the effect was inconsistent across different experiments, providing an example of genotype-by-environmental interaction.

Keywords: Grain weight, Grain size, Tightly-linked, Quantitative trait locus, Rice 


\section{INTRODUCTION}

51 Thousand grain weight (TGW) is a key component of grain yield in rice, and a trait closely

52 related to grain length $(\mathrm{GL})$ and grain width $(\mathrm{GW})$ that are important traits for grain quality.

53

These traits are all complex and inherited quantitatively. To date, causal genes for 16 quantitative trait loci (QTL) affecting these traits have been isolated. They are distributed across eight of the 12 chromosomes of rice. Two of them, GW7/GL7 and GS9 (Wang et al., 2015a; Wang et al., 2015b; Zhao et al., 2018), have large effect on grain shape but no effect on TGW due to opposite allelic directions of the effects on GL and GW. Four of them, GW2 (Song et al., 2007), GS5 (Li et al., 2011), GSE5 (Duan et al., 2017) and GW8 (Wang et al., 2012), have larger effects on TGW and GW than on GL. Six others, GL2/GS2 (Che et al., 2016; Hu et al., 2015), GS3 (Fan et al., 2006), GL3.1/qGL3 (Qi et al., 2012; Zhang et al., 2012), OsLG3 (Yu et al., 2017), qTGW3 (Hu et al., 2018) and GW6a (Song et al., 2015), have larger effects on TGW and GL than on GW. The remaining four, OsLG3b (Yu et al., 2018), GL4 (Wu et al., 2017), TGW6 (Ishimaru et al., 2013) and GLW7 (Si et al., 2016), have large effects on TGW and GL but no effect on GW. These genes encode proteins that are involved in regulating cell proliferation and elongation, including phytohormones, transcriptional regulatory factors and components of pathways such as ubiquitin-proteasome, G protein signaling and MAPK signaling (Li et al., 2018). These studies have substantially improved our understanding of the molecular basis of grain weight in rice, but the information is rather fragmental and more genes remain to be isolated and characterized (Zuo $\& L i, 2014)$.

In genetic analysis of agronomic traits, close linkage of QTL controlling the same trait has been frequently observed. Cloned QTL having major effects on grain weight and grain size in rice have provided clear examples. $O s L G 3$ and $O s L G 3 b$ for grain length (Yu et al., 2017; Yu et al., 2018) were located in a 1.7-Mb region of chromosome 3, GS5 and GSE5 for grain width in a 1.9-Mb region of chromosome 5 (Li et al., 2011; Duan et al., 2017), and TGW6 and GW6a for grain weight in a 0.6-Mb region of chromosome 6 (Ishimaru et al., 2013; Song et al., 2015). Similar results have also been reported for QTL having minor effects on these traits. Targeting at a QTL region affecting grain weight difference between two indcia rice varieties, six minoreffect QTL were separated in a 7.1-Mb interval on chromosome 1, including qTGW1.1a, qTGW1.1b, qTGW1.2a, qTGW1.2b, qGS1-35.2 and qGW1-35.5 (Wang et al., 2015c; Zhang et al., 2016; Dong et al., 2018). Assumably, validation and dissection of QTL that were previously 
81

coarsely mapped could provide a large number of candidates for isolating individual genes controlling grain weight and grain size in rice.

Rice is a staple food for about half of the world's population. Large scale cultivation of hybrid rice has made a great contribution to ensuring the food security in China and may make contributions in many other countries as well (Yuan, 2014; Nalley et al., 2016). Minor-effect QTL is believed to play a critical role in controlling genetic variations of yield traits among commercial rice varieties and the dissection of these QTL would facilitate the development of high-yielding rice varieties (Kinoshita et al., 2017). In our previous studies, QTL analysis for grain size was performed using three recombinant inbred line populations constructed from crosses between parental lines of commercial hybrid rice. One QTL, $q G S 10$ conferring grain size difference between Teqing (TQ) and IRBB lines was mapped in the interval RM6100-RM228 on chromosome 10 and then validated using near isogenic lines (NILs) of the same cross (Wang et al., 2017). In the present study, NIL populations segregated in sequential order around the $q$ GS10 region were constructed from the TQ/IRBB52 cross and used for QTL analysis. Three tightly-linked QTL were separated. Among them, $q$ GS10.1 had a stable and relatively high effect on grain weight, grain length and grain width, $q$ GS10.2 had significant effects on all the three traits but the effects appeared to be greatly influenced by genotype-by-environmental interaction, and $q G L 10.1$ was shown to affect grain length only.

\section{MATERIALS AND METHODS}

\section{Rice materials}

A total of 12 populations of rice (Oryza sativa L.) were used (Table 1). As illustrated in Fig. 1 and described below, these populations were derived from two residual heterozygous $(\mathrm{RH})$ plants of the indica rice cross TQ/IRBB52, namely ZC9 and Ti52-1. ZC9 was an F9 plant carrying four heterozygous segments, including RM6704-RM3773 on chromosome 10 and three others on chromosomes 1, 9 and 11 (Fig. 2). Ti52-1 was an $\mathrm{F}_{7}$ plant carrying one heterozygous segment, i.e., RM25766-RM228 on chromosome 10. The intervals RM6704-RM3773 and RM25766-RM228 are adjacent and jointly covered the RM6100-RM228 region previously mapped for qGS10 (Wang et al., 2017). Two populations were constructed and used for QTL mapping. One consisted of $203 \mathrm{~S}_{1: 2}$ families derived from ZC9, and the other comprised $171 \mathrm{~S}_{1}$ plants produced from Ti52-1 (Table 1).

The other ten populations used for QTL mapping were all derived from Ti52-1. Five $\mathrm{F}_{9}$ 
112 plants with sequential heterozygous regions covering RM25766-RM228 were selected from

113 progenies of Ti52-1. They were selfed to produce five new $\mathrm{S}_{1}$ populations. The number of plants

114 in each population ranged from 172 to 343 (Table 1). In each population, non-recombinant

115 homozygotes were selected and selfed. Five NIL populations in $\mathrm{F}_{\text {10:11 }}$ were established, in which

116 the numbers of NILs having the same genotype ranged from 28 to 38 (Table 1).

\section{Field trial and phenotypic evaluation}

118 The filed experiments were conducted at the experimental stations of the China National Rice

119 Research Institute located in either Hangzhou, Zhejiang Province, or Lingshui, Hainan Province, 120 China. The plants were grown with $16.7 \mathrm{~cm}$ between plants and $26.7 \mathrm{~cm}$ between rows.

121 The six $\mathrm{S}_{1}$ populations were grown without replication and the plants were harvested 122 individually. The remaining six populations, including one $\mathrm{S}_{1: 2}$ population and five NIL 123 populations, were tested using a randomized complete block design with two replications.

124 Twelve plants per line were planted in each row. The middle five plants were harvested.

125 Measurement of TGW, GL and GW in all the experiments followed the method of Zhang et al. 126 (2016).

\section{DNA marker analysis}

128 For the ZC9 population, marker data and genetic maps have been available (Sun et al., 2018).

129 For the other populations, a total of 12 DNA markers were used, including five simple sequence 130 repeat (SSR) and seven InDel markers. The SSRs were selected from the Gramene database 131 (www.gramene.org), while the InDels (Table 2) were developed based on the sequence 132 differences between TQ and IRBB52. DNA extraction and PCR amplification followed the 133 method of Zheng et al. (1995) and Chen et al. (1997), respectively.

\section{Data analysis}

Mapmarker/Exp 3.0 was performed to calculate the genetic distance which was presented in centiMorgan (cM) using the Kosambi function (Lander et al., 1987). For the six $\mathrm{S}_{1}$ populations and the ZC9 $\mathrm{S}_{1: 2}$ population, QTL analysis was performed with the inclusive composite interval mapping (ICIM) using the BIP (QTL mapping in the biparental populations) functionality of QTL IciMapping 4.1 (Meng et al., 2015). LOD thresholds were calculated with 1000 permutation tests $(P<0.05)$ and used to declare a putative QTL. For the five NIL populations, SAS procedure GLM (SAS Institute Inc 1999) was used to determine phenotypic differences in each population. A mixed model GENOTYPE + LINE (GENOTYPE) + REP + 
143

144

145

146

147

148

149

150

151

152

153

154

155

156

157

158

159

160

161

162

163

164

165

166

167

168

169

170

171

172

173

GENOTYPE*REP was applied, in which LINE (GENOTYPE) was defined as a random effect and used as the error term to test GENOTYPE differences (Dai et al., 2008). Additive effect and the proportion of phenotypic variance explained $\left(R^{2}\right)$ were estimated with the same model. QTL were designated following the rule proposed by McCouch and CGSNL (2008).

\section{RESULTS}

\section{QTL for grain size detected in the ZC9 and Ti52-1 populations}

The ZC9 $\mathrm{S}_{1: 2}$ population was segregated in the interval RM6704-RM3773 that covered the upper portion of the $q G S 10$ region, and in three other intervals (Fig. 2). All the four regions were found to have significant effects on one or two of the three grain-size traits analyzed (Table 3 ). In the interval RM6704-RM3773 on chromosome 10, the effects were significant on GL but nonsignificant on the other two traits. This QTL explained $9.00 \%$ of the phenotypic variance and the IRBB52 allele increased GL by $0.020 \mathrm{~mm}$. The $q G L 1$ and $q G W 1$ associated with RM12210 on chromosome 1 had $R^{2}$ values of 32.71 and $5.84 \%$, respectively, with the IRBB52 allele increased GL by $0.041 \mathrm{~mm}$ and decreased GW by $0.007 \mathrm{~mm}$. The $q T G W 9$ and $q G W 9$ in the interval RM219-RM1896 on chromosome 9 had $R^{2}$ values of 19.15 and $7.42 \%$, respectively, with the IRBB52 allele increased TGW by $0.16 \mathrm{~g}$ and GW by $0.007 \mathrm{~mm}$. The $q T G W 11$ and $q G W 11$ in the interval RM224-RM5926 on chromosome 11 had $R^{2}$ values of 13.81 and $31.69 \%$, respectively, with the IRBB52 allele decreased TGW by $0.14 \mathrm{~g}$ and GW by $0.016 \mathrm{~mm}$.

The Ti52-1 population was segregating in the interval RM25766-RM228 only, which covered the lower portion of the $q G S 10$ region (Fig. 2). This region was found to have significant effects on all the three grain-size traits analyzed (Table 3). The QTL effects contributed 20.15$36.58 \%$ to the phenotypic variance of each trait, with the TQ allele increasing TGW, GL and GW by $0.59 \mathrm{~g}, 0.081 \mathrm{~mm}$ and $0.024 \mathrm{~mm}$, respectively. Since $q G S 10$ was previously reported to affect all these three traits with the TQ allele always increasing trait values (Wang et al., 2017), it is concluded that $q G S 10$ was located in the segregating region of the Ti52-1 population, and another QTL, $q G L 10.1$, was located in an adjacent interval that was segregated in the ZC9 population (Fig. 3A).

\section{Dissection of $q$ GS10 into two QTL for grain size and grain weight}

For further validation and dissection of $q G S 10$, five $\mathrm{S}_{1}$ populations with sequential segregating regions covering the interval RM25766-RM228 were established (Table 1; Fig. 3B). Results of QTL analysis for TGW, GL and GW using these populations are shown in Table 4. Significant 
174 QTL effects were detected in G10-2, G10-4 and G10-5 but not in the other two populations. In

175 G10-2, the effects were significant for all the three traits. The TQ allele increased TGW, GL and

176 GW by $0.19 \mathrm{~g}, 0.030 \mathrm{~mm}$ and $0.004 \mathrm{~mm}$, respectively. In G10-4 and G10-5, significant QTL

177 effects were detected for GL only. The enhancing alleles were derived from IRBB52 in both

178 populations, with similar additive effects of 0.019 and $0.023 \mathrm{~mm}$, and similar $R^{2}$ values of 5.11

179 and $3.01 \%$. As shown in Fig. 3B, the region segregated in G10-2 was separated from those

180 segregated in G10-4 and G10-5, indicating that at least two QTL were responsible for the grain-

181 size variations in these populations. One QTL was located in the segregating region of G10-2

182 and another QTL may be located in the common segregating region of G10-4 and G10-5. To

183 validate these results, non-recombinant homozygotes of the five $\mathrm{S}_{1}$ populations were identified,

184 from which five NIL populations maintaining the original sequential segregating regions were

185 constructed (Table 1; Fig. 3B).

186 Distributions of TGW, GL and GW in the five NIL populations are shown in Fig. 4. The

187 three traits were continuously distributed in all the populations, but differentiation between the

188 two genotypic groups was observed in four populations. Concentration of the TQ and IRBB52

189 homozygous lines respectively in the high- and low-value regions was evident for TGW and GL

190 in G11-2, and for all the three traits in G11-3. The same tendency, though less obvious, was

191 found for TGW and GW in G11-4 and G11-5. Since the segregating region of G11-3 covered the

192 segregating regions of G11-2, G11-4 and G11-5 (Fig. 3B), these results suggest that allelic

193 differences of the two QTL, one located in the segregating region of G11-2 and the other in the

194 common segregating region of G11-4 and G11-5, could be the main cause of phenotypic

195 variations in the five NIL populations.

196 Results of two-way ANOVA for TGW, GL and GW using the five NIL populations are

197 presented in Table 5. In G11-1, no significant phenotypic difference was detected between the

198 two genotypic groups, matching well to the detection of no QTL in its origin population G10-1.

199 In G11-2, the two genotypic groups showed significant differences on each trait. The TQ allele

200 increased TGW, GL and GW by $0.21 \mathrm{~g}, 0.032 \mathrm{~mm}$ and $0.004 \mathrm{~mm}$, with $R^{2}$ values of $40.58,28.55$

201 and $3.99 \%$, respectively. These results were in general agreement with QTL detected in the

202 G10-2 population from which G11-2 was originated, confirming that a QTL affecting TGW, GL

203 and GW was located in the segregating region of G10-2 and G11-2. This QTL, designated

$204 q$ qS10.1, was mapped within the region flanked by InDel markers Te20811 and Te21018 (Fig. 
205

206

207

208

209

210

211

212

213

214

215

216

217

218

219

220

221

222

223

224

225

226

227

228

229

230

231

232

233

234

235

3B), corresponding to a 207.1-kb region in the Nipponbare genome.

In G11-4 and G11-5 which were segregated in homozygous regions of G11-2, significant QTL effects were detected for TGW and GW with the TQ allele always increasing the trait values. The additive effects were 0.13 and $0.12 \mathrm{~g}$ for TGW, and 0.012 and $0.011 \mathrm{~mm}$ for $\mathrm{GW}$. These results indicate that a QTL for TGW and GW was located in the common segregating region of G11-4 and G11-5. This QTL, designated qGS10.2, was mapped within the region flanked by SSR markers RM3123 and RM6673 (Fig. 3B), corresponding to a 1.2-Mb region in the Nipponbare genome. It is noted that the effect of $q$ GS10.2 on TGW and GW was not detected in the G10-4 and G10-5 populations from which G11-4 and G11-5 were originated. For the other trait GL, a small effect with the enhancing allele derived from IRBB52 was detected in G11-5, which was in accordance with the effects detected in G10-4 and G10-5.

For the remaining NIL population, G11-3, significant QTL effects with the enhancing allele derived from TQ were detected for all the three traits. The additive effects and $R^{2}$ values estimated for TGW and GW were higher in G11-3 than in G11-2, G11-4 and G11-5. This is reasonable because $q G S 10.1$ and $q G S 10.2$ have the same allelic direction and the segregating region of G11-3 covered both QTL.

\section{DISCUSSION}

Grain yield of $F_{1}$ hybrid rice are known to be positively correlated to both the $F_{1}$ heterosis and the parental values. Regarding phenotypic performances of the three key yield components, the highest correlation between restorer line (male parent) and $\mathrm{F}_{1}$ hybrid was found for grain weight (Xiang et al., 2016). Identification of QTL responsible for grain weight variation among important restorer lines would provide new information on understanding the genetic basis of grain yield in rice. In this study, QTL analysis using NILs derived from a cross between rice restorer lines TQ and IRBB52 resulted in the dissection of three QTL for grain weight and grain size that were tightly lined on the long arm of chromosome 10 , providing new candidates for gene cloning and marker assisted selection.

No QTL for grain weight and grain size detected on chromosome 10 have been cloned, but a number of studies (Ren et al., 2016; Qi et al. 2017; Okada et al., 2018) have located QTL for these traits in the $q G S 10$ region. In addition, this region was associated with grain-size difference not only between restorer lines TQ and IRBB52, but also between Zhenshan 97 and Milyang 46 (Wang et al., 2017) that were among the most important female and male parents of commercial 
236 hybrid rice, respectively. One of the three QTL for grain size separated in this region, $q$ GS10.1,

237 was shown to have a stable and relatively high effect on all the three grain-size traits analyzed.

238 Cloning of this QTL may identify an important gene controlling grain yield of modern rice 239 varieties.

240 Genotype-by-environmental interaction is an important factor influencing the performance 241 of complex traits. The effect of another QTL affecting all the three traits in this study, qGS10.2, 242 appeared to be inconsistent across different experiments. In the $S_{1}$ populations G10-3 and G10-4 243 tested in Lingshui, this QTL showed non-significant effect on TGW and GW (Table 4). In the 244 NIL populations G11-3 and G11-4 tested in Hangzhou, this QTL was detected for TGW and GW with the $R^{2}$ values ranging from 10.32 to $24.56 \%$ (Table 5). Since G11-3 and G11-4 were populations consisting of homozygous lines directly produced from G10-3 and G10-4, the discrepancy is unlikely caused by differences on the genetic background. Okada et al. (2018) also mapped a QTL for grain weight and grain width in this region, which had a large effect in the late-maturing sub-population but showed non-significant effect in the early-maturing subpopulation. They inferred that the difference was caused by the temperature difference from flowering to ripening between the two sub-populations, which was higher for the late-maturing sub-population. Similarly, a stronger effect of $O s M A D S 56 / q H d 1$ on grain weight was found to be associated with a higher air temperature (Chen et al., 2018). The average air temperature from flowering to ripening was $23.5^{\circ} \mathrm{C}$ for $\mathrm{G} 10-3$ and $\mathrm{G} 10-4$, and $27.2^{\circ} \mathrm{C}$ for $\mathrm{G} 11-3$ and $\mathrm{G} 11-4$. This could be the main cause for the inconsistent effect of GS10.2 observed in the present study.

Linkage drag is a problem commonly encountered in the introgression of alien genes. Suh et al. (2011) reported that lower spikelet fertility was associated with the introduction of the Bph8 resistance gene from $O$. australiensis into the background of a japonica rice variety. Yan et al. (2014) found that transformation of the $B t$ resistance gene into indica rice restorer line Minghui 63 may have negative effects on grain weight and grain size, resulting in lower grain yield. One of the parental lines used in the present study, IRBB52, is a rice restorer line carrying Xa4 and $X a 21$ in the genetic background of IR24. In the ZC9 population, three regions other than the targeting region on chromosome 10 were also segregated. QTL for grain size were detected in all these three regions (Table 3), among them $q T G W 11$ and $q G W 11$ were located in a region covering the Xa4 locus (Fig. 2). The IRBB52 allele decreased TGW and GW by $0.14 \mathrm{~g}$ and 266 $0.016 \mathrm{~mm}$. These results suggest that linkage drag may have occurred in the introgression of Xa4 
267 from the donor parent into IR24. Fine mapping of QTL in this region may provide a solution for 268 this problem.

\section{CONCLUSION}

270 Using NIL populations with sequential segregating regions jointly covering the entire interval for

271 QTL qGS10 mapped previously, three QTL for grain size tightly-linked on the long arm of

272 chromosome 10 were separated. One of them, $q$ GS10.1, had a stable and relatively high effect on

273 grain weight, grain length and grain width, providing a good candidate for gene cloning. Another

274 QTL, $q G S 10.2$, had a significant effect on all the three traits but the effect was inconsistent

275 across different experiments, providing an example of genotype-by-environmental interaction.

276

277

\section{ABBREVIATIONS}

278 TGW 1000-grain weight

279 GL grain length

280 GW grain width

281 QTL quantitative trait locus

282 TQ Teqing

283 NIL near isogenic line

284 SSR simple sequence repeat

285 GLM general linear model

286

ADDITIONAL INFORMATION AND DECLARATIONS

Funding

This work was supported by the National Key R\&D Program of China (2016YFD0101104), the National Natural Science Foundation of China (31521064), and a project of the China National Rice Research Institute (2017RG001-2).

294 The following grant information was disclosed by the authors:

295 National Key R\&D Program of China: 2016YFD0101104.

296 National Natural Science Foundation of China: 31521064.

297 Project of the China National Rice Research Institute: 2017RG001-2 
299 Competing Interests

300 The authors declare there are no competing interests.

301

302

\section{Author Contributions}

303

304

305

306

307

308

309

310

Jieyun Zhuang, Shaoqing Tang and Tongmin Mou conceived and designed the experiments. Yujun Zhu, Zhichao Sun, Xiaojun Niu and Jiezheng Ying performed the experiments.

Yujun Zhu, Yeyang Fan and Jieyun Zhuang analyzed the data.

Jieyun Zhuang and Yujun Zhu wrote the manuscript.

All authors read and approved the final manuscript.

\section{Data Availability}

The following information was supplied regarding data availability:

The raw data has been supplied as a Supplemental Information 1 and 2.

\section{REFERENCES}

Che R, Tong H, Shi B, Liu Y, Fang S, Liu D, Xiao Y, Hu B, Liu L, Wang H, Zhao M, Chu C. 2016. Control of grain size and rice yield by GL2-mediated brassinosteroid responses. Nature Plants 2: 1-7 DOI 10.1038/NPLANTS.2015.195.

Chen J-Y, Zhang H-W, Zhang H-L, Ying J-Z, Ma L-Y, Zhuang J-Y. 2018. Natural variation at $q H D 1$ affects heading date acceleration at high temperatures with pleiotropism for yield traits in rice. BMC Plant Biology 18: 112 DOI 10.1186/s12870-018-1330-5.

Chen X, Temnykh S, Xu Y, Cho YG, McCouch SR. 1997. Development of a microsatellite framework map providing genome-wide coverage in rice (Oryza sativa L.). Theoretical \& Applied Genetics 95: 553-567 DOI 10.1007/s001220050596.

Dai W, Zhang K, Wu J, Wang L, Duan B, Zheng K, Cai R, Zhuang J. 2008. Validating a segment on the short arm of chromosome 6 responsible for genetic variation in the hull silicon content and yield traits of rice. Euphytica 160: 317-324 DOI 10.1007/s10681-0079501-8. 
327

328

329

330

331

332

333

334

335

336

337

338

339

340

341

342

343

344

345

346

347

348

349

350

351

352

353

354

355

356

Dong Q, Zhang ZH, Wang LL, Zhu YJ, Fan YY, Mou TM, Ma LY, Zhuang JY. 2018. Dissection and fine-mapping of two QTL for grain size linked in a 460-kb region on chromosome 1 of rice. Rice 11: 44 DOI 10.1186/s12284-018-0236-z.

Duan P, Xu J, Zeng D, Zhang B, Geng M, Zhang G, Huang K, Huang L, Xu R, Ge S, Qian Q, Li Y. 2017. Natural variation in the promoter of GSE5 contributes to grain size diversity in rice. Molecular Plant 10: 685-694 DOI 10.1016/j.molp.2017.03.009.

Fan C, Xing Y, Mao H, Lu T, Han B, Xu C, Li X, Zhang Q. 2006. GS3, a major QTL for grain length and weight and minor QTL for grain width and thickness in rice, encodes a putative transmembrane protein. Theoretical \& Applied Genetics 112: 1164-1171 DOI 10.1007/s00122-006-0218-1.

Hu J, Wang Y, Fang Y, Zeng L, Xu J, Yu H, Shi Z, Pan J, Zhang D, Kang S, Zhu L, Dong G, Guo L, Zeng D, Zhang G, Xie L, Xiong G, Li J, Qian Q. 2015. A rare allele of GS2 enhances grain size and grain yield in rice. Molecular Plant 8: 1455-1465 DOI 10.1016/j.molp.2015.07.002.

Hu Z, Lu SJ, Wang MJ, He H, Sun L, Wang H, Liu XH, Jiang L, Sun JL, Xin X, Kong W, Chu C, Xue HW, Yang J, Luo X, Liu JX. 2018. A novel QTL $q T G W 3$ encodes the GSK3/SHAGGY-Like kinase OsGSK5/OsSK41 that interacts with OsARF4 to negatively regulate grain size and weight in rice. Molecular Plant 11: 736-749 DOI 10.1016/j.molp.2018.03.005.

Ishimaru K, Hirotsu N, Madoka Y, Murakami N, Hara N, Onodera H, Kashiwagi T, Ujiie K, Shimizu B, Onishi A, Miyagawa H, Katoh E. 2013. Loss of function of the IAAglucose hydrolase gene $T G W 6$ enhances rice grain weight and increase yield. Nature Genetics 45: 707-713 DOI 10.1038/ng.2612.

Kinoshita N, Kato M, Koyasaki K, Kawashima T, Nishimura T, Hirayama Y, Takamure I, Sato T, Kato K. 2017. Identification of quantitative trait loci for rice grain quality and yield-related traits in two closely related Oryza sativa L. subsp. japonica cultivars grown near the northernmost limit for rice paddy cultivation. Breeding Science 67: 191-206 DOI $10.1270 /$ jsbbs. 16155 .

Lander E, Green P, Abrahamson J, Barlow A, Daley M, Lincoln S, Newburg L. 1987. MAPMAKER: an interactive computer package for constructing primary genetic maps of 
357

358

359

360

361

362

363

364

365

366

367

368

369

370

371

372

373

374

375

376

377

378

379

380

381

382

383

384

385

386

experimental and natural populations. Genomics 1: 174-181 DOI 10.1016/08887543(87)90010-3.

Li Y, Fan C, Xing Y, Jiang Y, Luo L, Sun L, Shao D, Xu C, Li X, Xiao J, He Y, Zhang Q. 2011. Natural variation in GS5 plays an important role in regulating grain size and yield in rice. Nature Genetics 43: 1266-1269 DOI 10.1038/ng.977.

Li N, Xu R, Duan P, Li Y. 2018. Control of grain size in rice. Plant Reproduction 31: 237-251 DOI 10.1007/s00497-018-0333-6.

McCouch SR, CGSNL (Committee on Gene Symbolization, Nomenclature and Linkage, rice genetics cooperative). 2008. Gene nomenclature system for rice. Rice 1: 72-84 DOI 10.1007/s12284-008-9004-9.

Meng L, Li H, Zhang L, Wang J. 2015. QTL IciMapping: Integrated software for genetic linkage map construction and quantitative trait locus mapping in biparental populations. The Crop Journal 3: 269-283 DOI 10.1016/j.cj.2015.01.001.

Nalley L, Tack J, Barkley A, Jagadish K, Brye Kristofor. 2016. Quantifying the agronomic and economic performance of hybrid and conventional rice varieties. Crop Economics, Production \& Management 108: 1514-1523 DOI 10.2134/agronj2015.0526.

Okada S, Onogi A, Iijima K, Hori K, Iwata H, Yokoyama W, Suehiro M, Yamasaki M. 2018. Identification of QTLs for grain size using a novel set of chromosomal segment substitution lines derived from Yamadanishiki in the genetic background of Koshihikari. Breeding Science 68: 210-218 DOI 10.1270/jsbbs.17112.

Qi L, Sun Y, Li J, Su L, Zheng X, Wang X, Li K, Yang Q, Qiao W. 2017. Identify QTLs for grain size and weight in common wild rice using chromosome segment substitution lines across six environments. Breeding Science 67: 472-482 DOI 10.1270/jsbbs. 16082.

Qi P, Lin YS, Song XJ, Shen JB, Huang W, Shan JX, Zhu MZ, Jiang L, Gao JP, Lin HX. 2012. The novel quantitative trait locus GL3.1 controls rice grain size and yield by regulating Cyclin-T1:3. Cell Research 22: 1666-1680 DOI 10.1038/cr.2012.151.

Ren D, Rao Y, Huang L, Leng Y, Hu J, Mei L, Zhang G, Zhu L, Gao Z, Dong G, Guo L, Qian Q, Zeng D. 2016. Fine mapping identifies a new QTL for brown rice rate in rice (Oryza Sativa L.). Rice 9: 4 DOI 10.1186/s12284-016-0076-7.

SAS Institute Inc. 1999. SAS/STAT User's Guide. Cary: SAS Institute. 
387

388

389

390

391

392

393

394

395

396

397

398

399

400

401

402

403

404

405

406

407

408

409

410

411

412

413

414

415

416

417

Si L, Chen J, Huang X, Gong H, Luo J, Hou Q, Zhou T, Lu T, Zhu J, Shangguan Y, Chen E, Gong C, Zhao Q, Jing Y, Zhao Y, Li Y, Cui L, Fan D, Lu Y, Weng Q, Wang Y, Zhan Q, Liu K, Wei X, An K, An G, Han B. 2016. OsSPL13 controls grain size in cultivated rice. Nature Genetics 48: 447-456 DOI 10.1038/ng.3518.

Song XJ, Huang W, Shi M, Zhu MZ, Lin HX. 2007. A QTL for rice grain width and weight encodes a previously unknown RING-type E3 ubiquitin ligase. Nature Genetics 39: 623630 DOI $10.1038 / \mathrm{ng} 2014$.

Song XJ, Kuroha T, Ayano M, Furuta T, Nagai K, Komeda N, Segami S, Miura K, Ogawa D, Kmura T, Suzuki T, Higashiyama T, Yamasaki M, Mori H, Inukai Y, Wu J, Kitano H, Sakakibara H, Jacobsen SE, Ashikari M. 2015. Rare allele of a previously unidentified histone $\mathrm{H} 4$ acetyltransferase enhances grain weight, yield, and plant biomass in rice. Proceedings of the National Academy of Sciences of the United States of America 112: 76-81 DOI 10.1073/pnas.1421127112.

Suh J-P, Yang S-J, Jeung J-U, Pamplona A, Kim J-J, Lee J-H, Hong H-C, Yang C-I, Kim Y-G, Jena KK. 2011. Development of elite breeding lines conferring Bph18 gene-derived resistance to brown planthopper (BPH) by marker-assisted selection and genome-wide background analysis in japonica rice (Oryza sativa L.). Field Crops Research 120: 215-222 DOI 10.1016/j.fcr.2010.10.004.

Sun Z, Zhu Y, Chen J, Zhang H, Zhang Z, Niu X, Fan Y, Zhuang J. 2018. Minor-effect QTL for heading date detected in crosses between indica rice cultivar Teqing and near isogenic lines of IR24. The Crop Journal 6: 291-298 DOI 10.1016/j.cj.2018.01.002.

Wang S, Wu K, Yuan Q, Liu X, Liu Z, Lin X, Zeng R, Zhu H, Dong G, Qian Q, Zhang G, Fu X. 2012. Control of grain size, shape and quality by $O S S P L 16$ in rice. Nature Genetics 44: 950-955 DOI 10.1038/ng.2327.

Wang S, Li S, Liu Q, Wu K, Zhang J, Wang S, Wang Y, Chen X, Zhang Y, Gao C, Wang F, Huang H, Fu X. 2015a. The $O S S P L 16-G W 7$ regulatory module determines grain shape and simultaneously improves rice yield and grain quality. Nature Genetics 47: 949-954 DOI 10.1038/ng.3352.

Wang Y, Xiong G, Hu J, Jiang L, Yu H, Xu J, Fang Y, Zeng L, Xu E, Xu J, Ye W, Meng X, Liu R, Chen H, Jing Y, Wang Y, Zhu X, Li J, Qian Q. 2015b. Copy number variation at the GL7 locus contributes to grain size diversity in rice. Nature Genetics 47: 944-949 DOI 
418

419

420

421

422

423

424

425

426

427

10.1038/ng.3346.

Wang LL, Chen YY, Guo L, Zhang HW, Fan YY, Zhuang JY. 2015c. Dissection of $q T G W 1.2$ to three QTLs for grain weight and grain size in rice (Oryza sativa L.). Euphytica 202: 119-127 DOI 10.1007/s10681-014-1237-7.

Wang Z, Chen JY, Zhu YJ, Fan YY, Zhuang JY. 2017. Validation of $q$ GS10, a quantitative trait locus for grain size on the long arm of chromosome 10 in rice (Oryza sativa L.). Journal of Integrative Agriculture 16: 16-26 DOI 10.1016/S2095-3119(16)61410-7.

Wu W, Liu X, Wang M, Meyer RS, Luo X, Ndjiondjop MN, Tan L, Zhang J, Wu J, Cai H, Sun C, Wang X, Wing RA, Zhu Z. 2017. A single-nucleotide polymorphism causes smaller grain size and loss of seed shattering during African rice domestication. Nature Plants 3: 17064 DOI 10.1038/nplants.2017.64.

Xiang C, Zhang H, Wang H, Wei S, Fu B, Xia J, Li Z, Gao Y, Ye G. 2016. Dissection of heterosis for yield and related traits using populations derived from introgression lines in rice. The Crop Journal 4: 468-478 DOI 10.1016/j.cj.2016.05.001.

Yan B, Liu R, Li Y, Wang Y, Gao G, Zhang Q, Liu X, Jiang G, He Y. 2014. QTL analysis on rice grain appearance quality, as exemplifying the typical events of transgenic or backcrossing breeding. Breeding Science 64: 231-239 DOI 10.1270/jsbbs.64.231.

Yu J, Xiong H, Zhu X, Zhang H, Li H, Miao J, Wang W, Tang Z, Zhang Z, Yao G, Zhang Q, Pan Y, Wang X, Rashid MAR, Li J, Gao Y, Li Z, Yang W, Fu X, Li Z. 2017. Os $L G 3$ contributing to rice grain length and yield was mined by Ho-LAMap. BMC Biology 15: 28 DOI 10.1186/s12915-017-0365-7.

Yu J, Miao J, Zhang Z, Xiong H, Zhu X, Sun X, Pan Y, Liang Y, Zhang Q, Rehman RMA, Li J, Zhang H, Li Z. 2018. Alternative splicing of $O s L G 3 b$ controls grain length and yield in japonica rice. Plant Biotechnology Journal 16: 1667-1678 DOI 10.1111/pbi.12903.

Yuan L-P. 2014. Development of hybrid rice to ensure food security. Rice Science 21: 1-2 DOI 10.1016/S1672-6308(13)60167-5.

Zhang X, Wang J, Huang J, Lan H, Wang C, Yin C, Wu Y, Tang H, Qian Q, Li J, Zhang H. 2012. Rare allele of $O S P P K L 1$ associated with grain length causes extra-large grain and a significant yield increase in rice. Proceedings of the National Academy of Sciences of the United States of America 109: 21534-21539 DOI 10.1073/pnas.1219776110. 
448 Zhang HW, Fan YY, Zhu YJ, Chen JY, Yu SB, Zhuang JY. 2016. Dissection of the

$449 \quad q T G W 1.1$ region into two tightly-linked minor QTLs having stable effects for grain weight

450 in rice. BMC Genetics 17: 98 DOI 10.1186/s12863-016-0410-5.

451 Zhao DS, Li QF, Zhang CQ, Zhang C, Yang QQ, Pan LX, Ren XY, Lu J, Gu MH, Liu QQ.

452 2018. GS9 acts as a transcriptional activator to regulate rice grain shape and appearance

453 quality. Nature Communications 9: 1240 DOI 10.1038/s41467-018-03616-y.

454 Zheng KL, Huang N, Bennett J, Khush GS. 1995. PCR-based marker-assisted selection in rice

455 breeding. IRRI Discussion Paper Series No.12. Manila: International Rice Research

$456 \quad$ Institute.

457 Zuo J, Li J. 2014. Molecular genetic dissection of quantitative trait loci regulating rice grain

458 size. Annual Review of Genetics 48: 99-118 DOI 10.1146/annurev-genet-120213-092138. 
Figure 1

Development of the rice populations used in this study. NIL, near isogenic line 


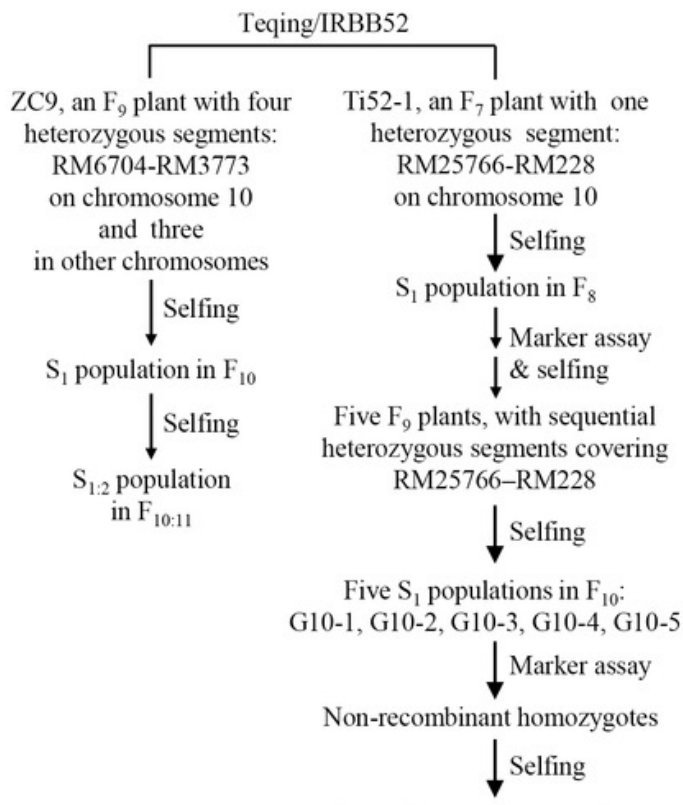

Five NIL populations in $\mathrm{F}_{10: 11}$ : G11-1, G11-2, G11-3, G11-4, G11-5 
Figure 2

Heterozygous regions of the residual heterozygous plants ZC9 and Ti52-1 


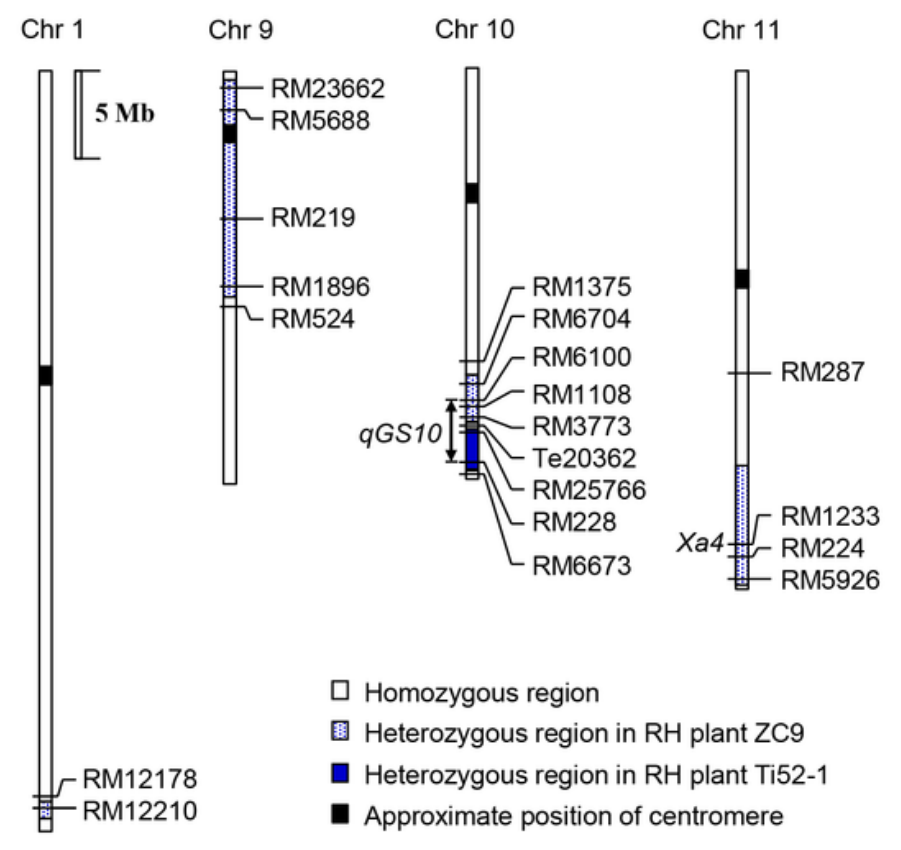


Figure 3

Genotypic composition of the rice populations in the target region on chromosome 10 


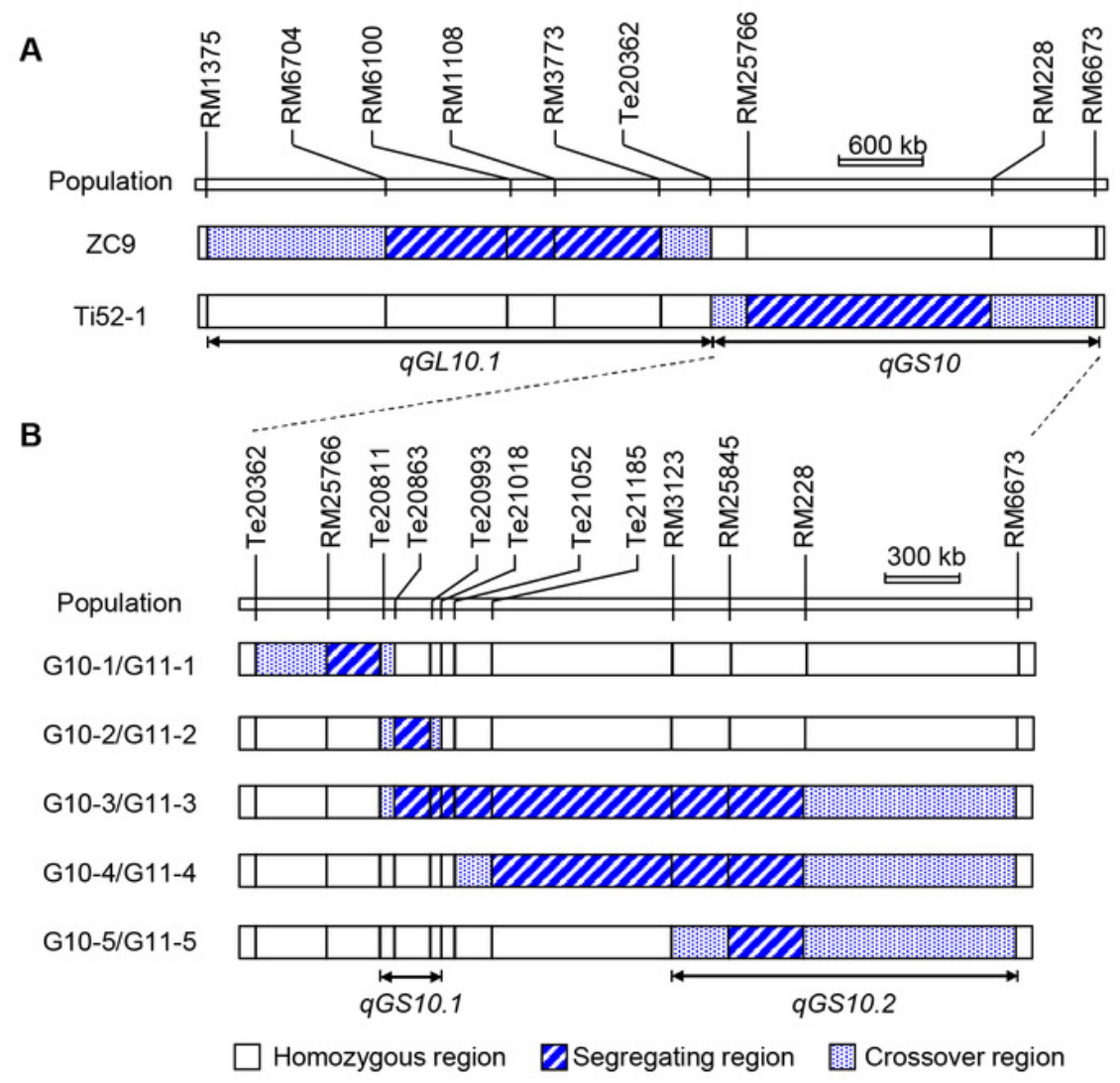


Figure 4

Distributions of 1000-grain weight, grain length and grain width in the five NIL populations 

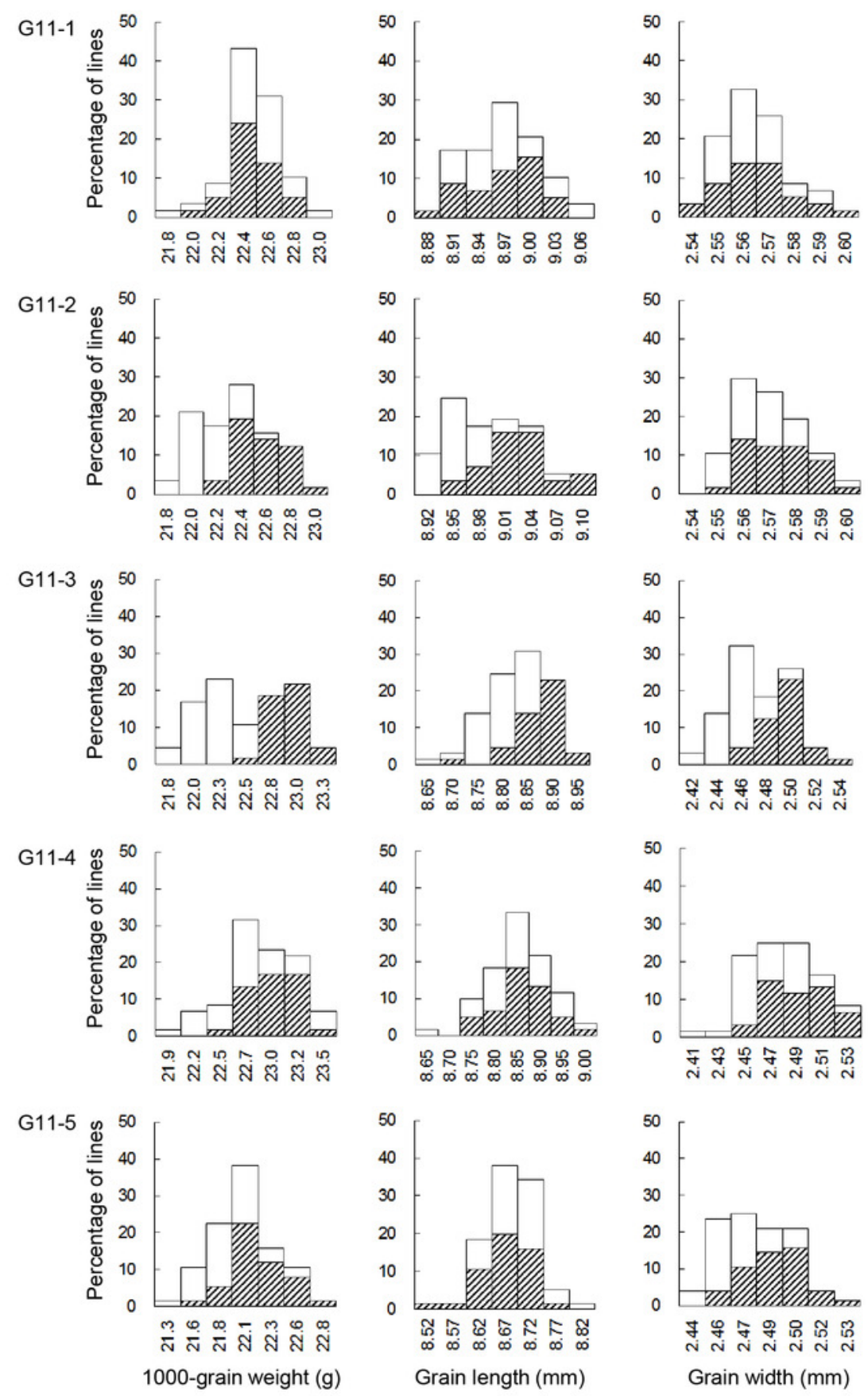

$\mathrm{NIL}^{\text {IRBB52 }}$ Ø NIL Teqing 
Table $\mathbf{1}$ (on next page)

Rice populations and field experiments 
1 Table 1 Rice populations and field experiments.

\begin{tabular}{|c|c|c|c|c|c|}
\hline \multicolumn{3}{|c|}{ Population } & \multirow{2}{*}{$\begin{array}{l}\text { Segregating regions } \\
\text { on chromosome } 10\end{array}$} & \multirow[t]{2}{*}{ Sample } & \multirow[t]{2}{*}{ Location and growth period } \\
\hline Name & Type & Generation & & & \\
\hline \multicolumn{6}{|c|}{ For validation of $G S 10$} \\
\hline ZC9 & $\mathrm{S}_{1: 2}$ & $\mathrm{~F}_{10: 11}$ & RM6704-RM3773 & 203 lines & HZ: May-Sep. 2017 \\
\hline Ti52-1 & $\mathrm{S}_{1}$ & $\mathrm{~F}_{8}$ & RM25766-RM228 & 171 plants & LS: Dec. 2015-Apr. 2016 \\
\hline \multicolumn{6}{|c|}{ For dissection of GS10 } \\
\hline G10-1 & $\mathrm{S}_{1}$ & $\mathrm{~F}_{10}$ & RM25766-Te20811 & 278 plants & LS: Dec. 2016-Apr. 2017 \\
\hline G10-2 & $\mathrm{S}_{1}$ & $\mathrm{~F}_{10}$ & Te20863-Тe20993 & 172 plants & LS: Dec. 2016-Apr. 2017 \\
\hline G10-3 & $\mathrm{S}_{1}$ & $\mathrm{~F}_{10}$ & Te20863-RM228 & 289 plants & LS: Dec. 2016-Apr. 2017 \\
\hline G10-4 & $\mathrm{S}_{1}$ & $\mathrm{~F}_{10}$ & Te21185-RM228 & 292 plants & LS: Dec. 2016-Apr. 2017 \\
\hline G10-5 & $\mathrm{S}_{1}$ & $\mathrm{~F}_{10}$ & RM25845-RM228 & 343 plants & LS: Dec. 2016-Apr. 2017 \\
\hline G11-1 & NIL & $\mathrm{F}_{10: 11}$ & RM25766-Te20811 & $29: 29$ lines & HZ: May-Sep. 2017 \\
\hline G11-2 & NIL & $\mathrm{F}_{10: 11}$ & Te20863-Te20993 & $29: 28$ lines & HZ: May-Sep. 2017 \\
\hline G11-3 & NIL & $\mathrm{F}_{10: 11}$ & Te20863-RM228 & $30: 35$ lines & HZ: May-Sep. 2017 \\
\hline G11-4 & NIL & $\mathrm{F}_{10: 11}$ & Te21185-RM228 & $30: 30$ lines & HZ: May-Sep. 2017 \\
\hline G11-5 & NIL & $\mathrm{F}_{10: 11}$ & RM25845-RM228 & $38: 38$ lines & HZ: May-Sep. 2017 \\
\hline
\end{tabular}

2 Notes.

3 NIL, near isogenic line; HZ, Hangzhou, Zhejiang Province; LS, Lingshui, Hainan Province. For the five NIL

4 populations, numbers of NILs carrying the Teqing and IRBB52 homozygous segments are indicated before and after 5 the colon, respectively. 
Table 2 (on next page)

Polymorphic markers developed and used in this study 
1 Table 2 Polymorphic markers developed and used in this study.

\begin{tabular}{lll}
\hline Name & Forward primer $\left(\mathbf{5}^{\prime} \mathbf{- 3} \mathbf{)}\right.$ & Reverse primer $\left(\mathbf{5}^{\prime} \mathbf{-} \mathbf{3}^{\prime} \mathbf{)}\right.$ \\
\hline Te20362 & TCTACCCTCACCTCGCGTA & TCCATTTCTAATCCACGACCT \\
Te20811 & ATGGAGAAGTAGCAGAGTACATT & TGGATCATCAAAGGCTCACAAA \\
Te20863 & GCCGCCTCTACGAGTT & AAAATCACCCGATTACCACAG \\
Te20993 & AAAATTTAACTTCTGCATGTTG & TCGAGCTTGCATGTCATC \\
Te21018 & GCTTCTAAACTGCTAACAGGT & GTCATTACAATTGCACATAGGAG \\
Te21052 & TCTGAATATTAGCATAGCCGAGT & CTTTTCCTGGGTATATGGAAC \\
Te21185 & ACCGCCGATGACATGC & TCTTCTTCGATCGGGTC \\
\hline
\end{tabular}

2 
Table 3 (on next page)

QTL for TGW, GL and GW detected in the ZC9 and Ti52-1 populations 
1 Table 3 QTL for TGW, GL and GW detected in the ZC9 and Ti52-1 populations.

\begin{tabular}{|c|c|c|c|c|c|c|c|c|}
\hline \multirow[t]{2}{*}{ Population } & \multicolumn{2}{|c|}{ Segregating region } & \multirow[t]{2}{*}{ Trait } & \multirow[t]{2}{*}{ QTL } & \multirow[t]{2}{*}{$L O D$} & \multirow[t]{2}{*}{$A$} & \multirow[t]{2}{*}{$D$} & \multirow[t]{2}{*}{$R^{2}(\%)$} \\
\hline & $\mathrm{Chr}$ & Interval & & & & & & \\
\hline \multirow[t]{7}{*}{ ZC9 } & 1 & RM12210 & $\mathrm{GL}$ & $q G L 1$ & 19.1 & 0.041 & -0.007 & 32.71 \\
\hline & & & $\mathrm{GW}$ & $q G W 1$ & 4.5 & -0.007 & -0.001 & 5.84 \\
\hline & 9 & RM219-RM1896 & TGW & $q T G W 9$ & 10.5 & 0.16 & -0.05 & 19.15 \\
\hline & & & GW & $q G W 9$ & 5.6 & 0.007 & -0.004 & 7.42 \\
\hline & 10 & RM6100-RM1108 & GL & $q G L 10.1$ & 6.1 & 0.020 & 0.003 & 9.00 \\
\hline & 11 & RM224-RM5926 & TGW & $q T G W 11$ & 7.8 & -0.14 & 0.023 & 13.81 \\
\hline & & & $\mathrm{GW}$ & $q G W 11$ & 20.2 & -0.016 & 0.003 & 31.69 \\
\hline \multirow[t]{3}{*}{ Ti52-1 } & 10 & RM25766-RM228 & TGW & $q T G W 10$ & 10.1 & -0.59 & -0.11 & 24.84 \\
\hline & & & GL & $q G L 10.2$ & 16.7 & -0.081 & 0.011 & 36.58 \\
\hline & & & GW & $q G W 10.2$ & 8.3 & -0.024 & -0.003 & 20.15 \\
\hline
\end{tabular}

2 Notes.

3 TGW, 1000-grain weight (g); GL, grain length (mm); GW, grain width (mm); Chr, chromosome; $A$, additive effect of

4 replacing a Teqing allele with an IRBB52 allele; $D$ : Dominance effect; $R^{2}$, proportion of phenotypic variance explained

5 by the QTL effect. QTL were designated following the rule proposed by McCouch and CGSNL (2008). 
Table 4 (on next page)

QTL for TGW, GL and GW detected in five $S_{1}$ populations 
1 Table 4 QTL for TGW, GL and GW detected in five $S_{1}$ populations.

\begin{tabular}{lllrlll}
\hline Population & Segregating region & Trait & LOD & $\boldsymbol{A}$ & $\boldsymbol{D}$ & $\boldsymbol{R}^{\mathbf{2}} \mathbf{( \% )}$ \\
\hline G10-1 & RM25766-Te20811 & TGW & n.s. & & & \\
& & GL & n.s. & & & \\
& & GW & n.s. & & & \\
G10-2 & Te20863-Te20993 & TGW & 14.6 & -0.19 & 0.66 & 32.09 \\
& & GL & 28.6 & -0.030 & 0.140 & 53.10 \\
& & GW & 8.5 & -0.004 & 0.035 & 20.12 \\
G10-3 & Te20863-RM228 & TGW & n.s. & & & \\
& & GL & n.s. & & & \\
& & GW & n.s. & & & \\
G10-4 & Te21185-RM228 & TGW & n.s. & & & \\
& & GL & 2.7 & 0.019 & 0.023 & 5.11 \\
& & GW & n.s. & & & \\
G10-5 & RM25845-RM228 & TGW & n.s. & & & \\
& & GL & 2.2 & 0.023 & -0.003 & 3.01 \\
& & GW & n.s. & & & \\
\hline
\end{tabular}

2 Notes.

3 TGW, 1000-grain weight (g); GL, grain length (mm); GW, grain width (mm); $A$, additive effect of replacing a Teqing 4 allele with an IRBB52 allele; $D$, dominance effect; $R^{2}$, proportion of phenotypic variance explained by the QTL effect; 5 n.s., not significant.

6 
Table 5 (on next page)

QTL for TGW, GL and GW detected in five NIL populations 
1 Table 5 QTL for TGW, GL and GW detected in five NIL populations.

\begin{tabular}{|c|c|c|c|c|c|c|c|c|}
\hline \multirow[t]{2}{*}{ Name } & \multirow[t]{2}{*}{ Segregating region } & \multirow[t]{2}{*}{ Trait } & \multicolumn{2}{|c|}{ Phenotypic mean } & \multirow[t]{2}{*}{$P$} & \multirow[t]{2}{*}{$A$} & \multirow[t]{2}{*}{$R^{2}(\%)$} & \multirow[t]{2}{*}{ QTL segregated } \\
\hline & & & $\overline{\text { NIL }^{T Q}}$ & NIL $^{\text {IRBB52 }}$ & & & & \\
\hline \multirow[t]{3}{*}{$\overline{\mathrm{G} 11-1}$} & RM25766-Te20811 & TGW & $22.37 \pm 0.18$ & $22.36 \pm 0.25$ & 0.9593 & & & No QTL \\
\hline & & GL & $8.953 \pm 0.041$ & $8.956 \pm 0.044$ & 0.7746 & & & \\
\hline & & GW & $2.560 \pm 0.015$ & $2.559 \pm 0.011$ & 0.6110 & & & \\
\hline \multirow[t]{3}{*}{ G11-2 } & Te20863-Te20993 & TGW & $22.46 \pm 0.21$ & $22.03 \pm 0.19$ & $<0.0001$ & -0.21 & 40.58 & $q G S 10.1$ \\
\hline & & GL & $9.009 \pm 0.041$ & $8.946 \pm 0.038$ & $<0.0001$ & -0.032 & 28.55 & \\
\hline & & GW & $2.568 \pm 0.013$ & $2.561 \pm 0.012$ & 0.0309 & -0.004 & 3.99 & \\
\hline \multirow[t]{3}{*}{ G11-3 } & Te20863-RM228 & TGW & $22.80 \pm 0.16$ & $22.06 \pm 0.23$ & $<0.0001$ & -0.37 & 63.80 & $q G S 10.1+q G S 10.2$ \\
\hline & & GL & $8.845 \pm 0.046$ & $8.767 \pm 0.045$ & $<0.0001$ & -0.039 & 27.34 & \\
\hline & & GW & $2.485 \pm 0.019$ & $2.446 \pm 0.018$ & $<0.0001$ & -0.020 & 40.55 & \\
\hline \multirow[t]{3}{*}{ G11-4 } & Te21185-RM228 & TGW & $22.89 \pm 0.25$ & $22.63 \pm 0.41$ & $<0.0001$ & -0.13 & 17.59 & $q G S 10.2$ \\
\hline & & GL & $8.839 \pm 0.058$ & $8.823 \pm 0.075$ & 0.4628 & & & \\
\hline & & GW & $2.483 \pm 0.021$ & $2.460 \pm 0.024$ & $<0.0001$ & -0.012 & 24.56 & \\
\hline \multirow[t]{3}{*}{ G11-5 } & RM25845-RM228 & TGW & $22.04 \pm 0.27$ & $21.80 \pm 0.28$ & $<0.0001$ & -0.12 & 10.32 & $q G S 10.2$ \\
\hline & & GL & $8.645 \pm 0.054$ & $8.664 \pm 0.046$ & 0.0356 & 0.010 & 2.29 & \\
\hline & & GW & $2.480 \pm 0.017$ & $2.459 \pm 0.017$ & $<0.0001$ & -0.011 & 17.51 & \\
\hline
\end{tabular}

\section{Notes.}

3 TGW, 1000-grain weight (g); GL, grain length (mm); GW, grain width (mm); $A$, additive effect of replacing a Teqing allele with

4 an IRBB52 allele; $R^{2}$, proportion of phenotypic variance explained by the QTL effect. NIL ${ }^{\mathrm{TQ}}$ and NIL ${ }^{\mathrm{IRBB} 52}$ are near-isogenic lines with Teqing and IRBB52 homozygous genotypes in the segregating region, respectively. 